\title{
Riso amaro? L'umorismo come rimedio contro il razzismo in Lakhous, Wadia e de Caldas Brito
}

\section{Maria Cristina Mauceri}

\section{(2) OpenEdition}

1 Journals

\section{Edizione digitale}

URL: https://journals.openedition.org/cher/11078

DOI: 10.4000/cher.11078

ISSN: 2803-5992

\section{Editore}

Presses universitaires de Strasbourg

\section{Edizione cartacea}

Data di pubblicazione: 30 juin 2013

Paginazione: 69-82

ISBN: 978-2-35410-054-4

ISSN: 1968-035X

Notizia bibliografica digitale

Maria Cristina Mauceri, «Riso amaro? L'umorismo come rimedio contro il razzismo in Lakhous, Wadia e de Caldas Brito», reCHERches [Online], 10 | 2013, online dal 08 février 2022, consultato il 10 février 2022. URL: http://journals.openedition.org/cher/11078 ; DOI: https://doi.org/10.4000/cher.11078

\section{(c) (i) (ㅇ)}

Ce(tte) œuvre est mise à disposition selon les termes de la Licence Creative Commons Attribution Pas d'Utilisation Commerciale - Partage dans les Mêmes Conditions 4.0 International. 


\title{
Riso amaro? \\ L'umorismo come rimedio contro il razzismo in Lakhous, Wadia e de Caldas Brito
}

\author{
Maria CRIStina MAuCERI \\ University of Sydney
}

U no dei personaggi di Scontro di civiltà per un ascensore a Piazza Vittorio di Amara Lakhous osserva che il razzista è una persona che non sorride mai e Amedeo/Ahmed, il protagonista del romanzo, dichiara: "Medici di tutto il mondo unitevi! Inventate un nuovo rimedio per curare i razzisti dall'invidia e dall'odio ${ }^{1}$. In effetti l'umorismo può avere una importante funzione sociale ed essere un mezzo efficace per denunciare e combattere una questione seria come il razzismo. In questo saggio analizzerò una scelta di testi di tre scrittori migranti, Amara Lakhous, Laila Wadia e Christiana de Caldas Brito, in cui l'umorismo serve per ridicolizzare il razzismo e la xenofobia degli italiani². Mi propongo di evidenziare i procedimenti e le tecniche narrative adoperati da questi autori. La mia analisi si basa sulle opere di diversi studiosi dell'umorismo, in particolare Henri Bergson, che

1 A. Lakhous, Scontro di civiltà per un ascensore a Piazza Vittorio, Roma, Edizioni e/o, 2006, p. 73. (D'ora in poi il testo sarà citato con la sigla SCAPV seguita dal numero della pagina).

2 Questo saggio non prende in considerazione le divertenti e argute vignette dello scrittore Kossi Komla-Ebri contenute nei due volumi Imbarazzismi. Quotidiani imbarazzi in bianco e nero, Milano, Edizioni dell'Arco, 2002 e Nuovi Imbarazzismi. Quotidiani imbarazzi in bianco e nero... e a colori, Milano, Edizioni dell'Arco Marna, 2004 in quanto sono già state oggetto di esaurienti analisi, cfr. M. Orton, Comedy and comunity, http:// www.kossi-komlaebri.net/Marie-Orton_Kossi-on-comedy.pdf e M. Purpura, «Stereotipi e imbarazzismi» in L'immaginario africano/italiano negli scrittori migranti, Tesi di laurea in Sociologia della Letteratura, a.a. 2003-2004. http://www.kossikomlaebri.net/ tesi_laurea_marco_purpura.php 
ha sottolineato il potere del riso come correttivo sociale, mentre Vladimir Propp ha evidenziato come il comico possa rivelare difetti palesi o nascosti ${ }^{3}$. Anche Sigmund Freud si è interessato all'umorismo, che considerava un meccanismo di difesa grazie al quale una persona evita di provare emozioni spiacevoli, ed anche una specie di sfida specialmente quando bersaglio dell'umorismo sono persone che rappresentano l'autoritàt. Infine la teoria di Luigi Pirandello dell'umorismo come «il sentimento del contrario» ${ }^{5}$ e l'importanza che questo scrittore attribuisce alla riflessione come attività della mente, che ci fa comprendere le cause del riso, si è rivelata utile per la mia analisi.

Scontro di civiltà per un ascensore a piazza Vittorio è una satira sulle difficoltà di Roma, ma il discorso si può estendere a tutta l'Italia, di convivere con gli immigrati e sui luoghi comuni e l'ignoranza che impediscono di accettare la diversità. Già il titolo è divertente perché l'inizio roboante, lo Scontro di civiltà, richiama lo studio di Samuel P. Huntington (1993) Clash of Civilizations and the Remaking of the new World Order in cui lo studioso americano fa riferimento ai conflitti a livello globale causati da divergenze culturali e religiose. Lakhous, invece, concentra lo scontro su uno spazio minimo come un ascensore e lo localizza in un condominio nel quartiere romano multietnico di Piazza Vittorio. A ben vedere non si tratta tanto di uno «scontro di civiltà» ma di una commedia degli equivoci, dovuta all'incapacità dei vari personaggi di comprendersi e di dialogare tra di loro. Il condominio è lo scenario e il laboratorio ideale per illustrare i problemi della convivenza multietnica, un esempio del 'nuovo ordine' che si sta creando in Italia con l'arrivo degli immigrati.

Il ritrovamento di un condomino ucciso nell'ascensore, spazio che come vedremo ha una valenza simbolica, dà l'avvio alle indagini e alle deposizioni dei diversi personaggi che sono interessanti non per scoprire il vero assassino (ognuno ha un'opinione diversa) ma perché rivelano la realtà di quel microcosmo etnico che è il quartiere intorno a Piazza Vittorio .

3 H. Bergson, Le rire. Essai sur la signification du comique, Paris, Alcan, 1924; V. J. Propp, Comicità e riso. Letteratura e vita quotidiana, Torino, Einaudi, 1988, p. 32.

4 S. Freud, «Der Humor» (1927) in Gesammelte Werke, 14. Band, Werke aus den Jahren 1925-1931, Frankfurt am Main, Fischer Verlag, 1976, p. 383-389.

5 L. Pirandello, L'umorismo, Milano, Mondadori, 1986, p. 135.

6 Per un'analisi dei diversi significati dei personaggi migranti e la funzione dello spazio nel romanzo si veda G. Parati, «Where Do Migrants Live? Amara Lakhous's Scontro di civiltà per un ascensore a Piazza Vittorio» in Annali di Italianistica. Capital City: Rome 1870-2010, 28, 2010. 
I vari personaggi nativi e stranieri, che si succedono sulla scena, raccontano la loro versione dei fatti da cui emergono le loro frustrazioni e il loro atteggiamento quasi sempre xenofobo verso chi è culturalmente diverso anche se non necessariamente proviene dall'estero. I personaggi italiani sono i più comici, quasi ridotti a macchiette ${ }^{7}$, perché Lakhous li presenta come caricature, esagerando alcuni dei loro tratti caratteriali per metterne a nudo i difetti, come è tipico di questo procedimento umoristico ${ }^{8}$.

La prima italiana ad apparire sulla scena è la custode del palazzo e dell'ascensore oggetto di discordia, la portinaia napoletana Benedetta Esposito. È una donna semplice e conservatrice, infatti difende fieramente Andreotti che per lei è un santo. Lakhous ci presenta una figura stereotipizzata che ha introiettato tutti i cliché sugli immigrati, tra cui quelli che rubano il lavoro agli italiani, portano malattie e sono tutti delinquenti (SCAPV, $47,48,51)$, stereotipi che nascono dalla paura e dall'ignoranza. La donna non sa distinguere tra i diversi gruppi etnici, anzi sembra trovar gusto a irritare gli stranieri del suo palazzo non attribuendo loro mai la giusta identità nazionale, ad esempio l'iraniano Parviz è un albanese, mentre la badante Maria Cristina deve essere per forza filippina anche se viene dal Perù. Lakhous non evidenzia solo l'ignoranza della donna, ma anche la sua visione riduttiva delle diverse comunità etniche che è segno di indifferenza e mancanza di rispetto.

Attraverso la mentalità di Benedetta, Lakhous fa anche capire come le persone semplici e ignoranti siano facilmente influenzabili dai media che, come è noto, hanno un ruolo importante nel diffondere pregiudizi e paure nei confronti degli immigrati. Benedetta cita le affermazioni sugli immigrati di Emilio Fede e Bruno Vespa come se fossero l'unica verità e per lei la diversità è un ostacolo insormontabile alla convivenza:

Vivere con loro è impossibile. Tengon religioni, abitudini e tradizioni diverse dalle nostre. Nei loro paesi vivono all'aperto o dentro le tende, mangiano con le mani, si spostano con i ciucci e i cammelli e trattano le donne come schiave. Io non sono razzista ma questa è la verità. Lo dice pure Bruno Vespa. (SCAPV, 50)

Benedetta è anche comica nel modo di parlare: l'italiano standard in cui si esprime è intercalato con colorite espressioni napoletane che rivelano le

7 M. G. Negro, «L'upupa o l'Algeria perduta: i nuclei tematici, il processo di riscrittura e la ricezione nel mondo arabo di Amara Lakhous» in Kumá. Creolizzare l'Europa, n. 12, 2006. http://www.disp.let.uniroma1.it/kuma/kuma12.html

8 V. Propp, op. cit., p. 77. 
sue origini meridionali. Ad esempio usa «guagliò» per rivolgersi all'iraniano Parviz, che pensa sia un insulto. L'equivoco genera comicità anche nel caso inverso, quando Benedetta crede che il «merci» che le rivolge Parviz, e che nella lingua persiana significa "grazie», sia una parolaccia.

L'avversione di Benedetta verso i migranti è motivata anche da fattori personali. Anche lei è un'immigrata, seppur 'interna', ma non ha nessuna simpatia per i nuovi immigrati che le ricordano il suo passato in quanto vittima a sua volta di pregiudizi e - come osserva la Parati - sfoga le sue frustrazioni sugli ultimi arrivati, per giunta stranieri9. In questo modo Lakhous ci ricorda che l'ostilità verso coloro che sono considerati diversi non è un fenomeno nuovo per l'Italia, ma l'unica differenza è il luogo di provenienza degli immigrati.

Un altro aspetto comico di Benedetta riguarda il suo atteggiamento verso il suo lavoro, infatti si considera la custode del palazzo e dell'ascensore oggetto della discordia. L'ascensore è un simbolo dello spazio a cui lei, come un poliziotto alla frontiera, vuole impedire l'accesso agli stranieri e, nello stesso tempo, è anche simbolo dello spazio non molto ampio che gli italiani devono imparare a condividere con gli stranieri ${ }^{10}$. L'alta considerazione che la donna ha di sé come custode degli spazi comuni del palazzo rivela il suo senso di superiorità verso i nuovi arrivati e il piacere che prova a esercitare il suo 'piccolo' potere su di loro. La comicità nasce dal contrasto tra il suo ruolo umile di portinaia e la sua illusione di sentirsi importante perché tiene a bada gli stranieri.

L'atteggiamento xenofobo non dipende dalla classe sociale delle persone, come dimostra un'altra figura razzista del romanzo, Elisabetta Fabiani, signora snob che vive nel palazzo ed è proprietaria di un cane misteriosamente scomparso. In questo caso l'esagerazione ha un ruolo rilevante nel rendere comica questa figura. Elisabetta nutre un affetto eccessivo e ossessivo per il suo cane e reagisce in modo esagerato alla sua scomparsa. È una figura autoreferenziale che vive chiusa nel suo mondo «animale» e la sua rigidità la rende comica ${ }^{11}$.

L'altro procedimento umoristico che Lakhous adopera per rendere ridicola Elisabetta è un'inversione, più precisamente un sovvertimento dei valori, per cui nella vita di questa donna l'oggetto della tolleranza non

9 G. Parati, «Where Do Migrants Live? Amara Lakhous's Scontro di civiltà per un ascensore a Piazza Vittorio», cit., p. 435.

10 Per i diversi significati dell'ascensore si rimanda al saggio della Parati.

11 Sugli effetti comici della rigidità cfr. H. Bergson, op. cit., p. 140. 
sono gli altri esseri umani, bensì i cani. Per Elisabetta i razzisti sono quelli che non rispettano «i diritti naturali e legittimi» di questi animali. «Io dico che prima vengono i diritti degli autoctoni, e i cani sono figli di questo paese» (SCAPV, 79) esclama indignata del fatto che gli immigrati facciano manifestazioni per il diritto al lavoro e all'alloggio.

È interessante notare che l'eccessiva cura degli italiani per i cani è un tema che ritroviamo in alcuni testi degli scrittori migranti ${ }^{12}$ che evidentemente provengono da culture in cui gli animali sono considerati creature impure o non sono stati innalzati a compagni dell'uomo come osserva l'iraniano Parviz che ricorda a Elisabetta che il compito dei cani è fare la guardia dai ladri, e si vede non solo chiamato zingaro ma anche tacciato di razzismo.

Il motivo del cane ha anche un'altra importante funzione nel romanzo. La narrazione assume toni paradossali e sconfina nel comico assurdo quando Elisabetta si lancia in un sproloquio anti-immigranti e propone di sostituirli con cani debitamente allenati. Elencando le diverse mansioni in cui gli immigrati possono essere sostituiti, indirettamente, la donna rivela come la loro presenza sia importante nella società italiana. In questo modo Lakhous dimostra quanto sia incongruente l'affermazione della donna che l'Italia non abbia bisogno di loro. Una delle teorie dell'umorismo si basa sul fattore dell'incongruenza, per cui il riso è una reazione a qualcosa di inaspettato, illogico e incoerente ${ }^{13}$. In effetti la comicità delle dichiarazioni di Elisabetta Fabiani nasce da un'incongruenza e dalla contraddizione che noi percepiamo tra quanto la donna afferma e la realtà che poi emerge dal suo discorso assurdo.

Benedetta ed Elisabetta sono caricature, ma gli aspetti umoristici di questi due personaggi sono mitigati dalle osservazioni di Amedeo/Ahmed, la figura centrale del romanzo, che da una parte funge da catalizzatore della narrazione, in quanto è stato accusato dell'uccisione di uno dei condomini e, dall'altra, è la sola persona in grado di spiegare cosa stia succedendo, perché con la sua attività di traduttore e mediatore culturale sa comprendere sia gli italiani sia gli stranieri. L'umorismo di Lakhous diventa tragicomico quando Ahmed spiega i motivi del razzismo di Benedetta e di Elisabetta.

Benedetta ha paura di perdere il suo lavoro perché l'ascensore si rompe spesso, mentre l'adorato cane di Elisabetta rappresenta un surrogato per il

12 Si vedano A. Malek Smari, L'occidentalista, Milano, Libribianchi, 2009 e O. Vorpsi, La mano che non mordi, Torino, Einaudi, 2007.

13 A. Koestler, The Act of Creation, New York, Macmillan, 1964, p. 35. 
figlio che l'ha abbandonata. Come aveva osservato Pirandello, l'umorismo nasce dal «sentimento del contrario». In effetti, prima ridiamo di Benedetta ed Elisabetta, poi l'impressione comica iniziale cambia poiché, attraverso le rivelazioni di Ahmed, capiamo che il loro comportamento nasce da paure e frustrazioni, per cui proviamo compassione per loro.

Il condominio è lo spazio ideale per mettere a confronto la convivenza tra autoctoni e stranieri anche nel romanzo Amiche per la pelle di Laila Wadia ${ }^{14}$ che racconta le vicissitudini per integrarsi di un gruppo di donne straniere di origini diverse che vivono con le loro famiglie in una casa del centro storico di Trieste. L'unico italiano che vi abita ancora, il Signor Rosso, è un concentrato di scorrettezza politica, infatti per lui tutti gli stranieri sono «negri», anche i cinesi, che hanno un'ulteriore aggravante come si può notare da questa citazione: «Pensavo che solo i vicentini erano mania gatti. Ora so che anche voi sporchi negri mangiate gatti per sfamare i milioni di musi neri che mettete al mondo come conigli» (AP, 14). È interessante il modo in cui Wadia gioca con uno stereotipo italiano e in questo modo fa vedere come oggetto di pregiudizio non siano solo i cinesi arrivati da poco, ma anche gli abitanti di una città italiana del settentrione.

Per ridicolizzare questo personaggio razzista Wadia ricorre a diversi procedimenti che riguardano l'aspetto fisico e il suo carattere. Secondo le componenti della comicità individuate da Bergson, due caratteristiche del Signor Rosso lo rendono comico: la misantropia e la rigidità che denota l'incapacità di guardarsi intorno ${ }^{15}$. Il Signor Rosso vive completamente isolato e l'unico oggetto del suo affetto sono i numerosi gatti randagi del quartiere di cui si prende cura. Inoltre è avaro, ad esempio si lamenta di dover pagare la stessa quota di consumo dell'acqua delle altre famiglie straniere del palazzo che invece sono numerose, e teme sempre che gli si possano chiedere dei soldi. Fin da Plauto l'avaro è un personaggio che viene spesso presentato in chiave comica. Il signor Rosso che vive chiuso al mondo esterno e si preoccupa solo di sé e di difendersi da quello che lui considera un'invasione di «negri» rivela un atteggiamento simile a quello di Benedetta Esposito nel romanzo di Lakhous, entrambi sono arroccati in posizione di difesa nei confronti dei migranti considerati pericolosi invasori del loro paese. Ė interessante notare come nel romanzo di Wadia e in quello di Lakhous, il personaggio italiano tratti gli animali come fossero persone,

14 L. Wadia, Amiche per la pelle, Roma, Edizioni e/o, 2007. (AP).

15 H. Bergson, op. cit., p. 149. 
mentre non ha lo stesso rispetto e cura verso altri esseri umani anche se di origine diversa.

Wadia usa una blanda animalizzazione del personaggio per ridicolizzarlo, «sembra un piccione spennato», osserva Shanti, l'indiana, io narrante del romanzo, che così ricorda il suo primo incontro con lui:

Aveva il riporto grigio che gli penzolava dalla parte sbagliata come un topo morto. Ho fatto un sussulto e per un momento ho pensato che fosse pazzo. «Cazzo, altri neri», ha borbottato.

Mi trovavo in Italia da pochi giorni e non capivo bene la lingua, per di più ero giovane ed ingenua. «Io mi chia-mo Shan-ti Ku-mar», gli ho risposto, scandendo le parole e allungando la mano. «Mio marito è Ash-ok Kumar. Abit-teremo terzo piano. Piacere di co-no-scer-la, Signor Cazzo Altrineri». $(\mathrm{AV}, 17)$

In questa scena l'umorismo non è solo legato al carattere e all'aspetto fisico del personaggio razzista, ma nasce anche dall'equivoco linguistico dovuto alla ancor scarsa conoscenza dell'italiano da parte dell'indiana. L'insulto razzista che l'uomo rivolge agli immigrati è preso per il suo nome. L'equivoco genera un'inversione che risulta comica per cui l'insulto verso lo straniero viene a ritorcersi contro l'italiano e lo fa ammutolire.

Mentre i personaggi razzisti di Lakhous restano chiusi nei loro monologhi autoreferenziali, il Signor Rosso, anziano e solo, riesce poi ad aprirsi ai suoi deprecati vicini e a intenerirsi per Kamla la figlia di Shanti, diventando per lei un nonno adottivo e un mediatore culturale perché le insegna l'italiano. A proposito dell'effetto curativo della risata, a cui fa riferimento Lakhous, sarà proprio una sana risata a segnare il momento in cui il signor Rosso inizia ad aprirsi agli stranieri. La risata sarà provocata dall'ingenua Kamla per la quale Ungaretti è solo una piazza e non un grande poeta.

In Amiche per la pelle c'è un'altra persona anziana che, apparentemente curiosa verso gli stranieri, si rivela poi razzista. Si tratta di una vecchia triestina che i protagonisti del romanzo incontrano su un autobus mentre stanno andando lungo la strada costiera dove c'è la sede dell'Istituto Internazionale di Fisica. L'anziana, descritta come «una strana creatura, metà donna e metà barboncino», quindi anche lei gentilmente animalizzata, si rivolge al marito pensando che le donne straniere e le loro famiglie appartengano alla comunità di scienziati che lavorano all'Istituto:

«Te digo mi, Paolo, questi forestieri ga delle teste. Un giorno o l'altro comanderanno lori».

Suo marito, da dietro il quotidiano, si è fatto il segno della croce. 
«Mica come quei altri la zò!», ha aggiunto con disprezzo la vecchia signora. Seguendo il suo sguardo abbiamo visto due distinti signori di colore seduti in ultima fila.

"Voi se studiati. A noi va ben gaver gente istruida qua. Volemo gente brava, non vu cumprà. Voi vi lavè, quei altri spuzza. Porta malattie, bestie». Mimava le sue parole, tappandosi il naso.

«Dai, basta, Lidia, lascia in pace le persone», le ha ordinato secco il marito, risistemandosi il collo della camicia bianca a quadretti sotto la giacca grigia. «Perché?», ha chiesto la signora anziana, stupita. «Me piazi parlar con la gente de fora. Me piazi saver come che i vivi. [...] Beh, go sentido dir che nel vostro paese maniè can. Fè di tutto col can: polpette, sugo, calandraca». (AP, 90)

L'umorismo di questa scena si basa sull'equivoco da cui emergono $\mathrm{i}$ pregiudizi della donna che associa al colore della pelle solo aspetti negativi. Le parole dell'anziana rivelano anche la sua ignoranza: si ritiene curiosa e interessata agli stranieri, ma poi non sa altro che ripetere cliché su di loro e l'incongruenza del suo modo di pensare ha un effetto umoristico. Va anche notata una certa teatralità della scena, specialmente nei gesti, come il segno della croce con cui il marito reagisce al pensiero che gli stranieri un giorno governeranno l'Italia e il tapparsi del naso della vecchietta, con cui rinforza mimicamente il suo pregiudizio che le persone di colore emettono cattivo odore, gesti che conferiscono comicità al personaggio. L'uso del dialetto triestino conferisce vivacità alla vecchietta, che però non si pone il minimo dubbio che $\mathrm{i}$ «forestieri» possano non comprenderla.

Mentre il signor Rosso, almeno inizialmente, appare decisamente razzista e riduce tutti gli stranieri al comune denominatore di «negri», la vecchietta triestina vorrebbe sembrare più aperta, ma in fondo anche lei è profondamente ignorante e razzista verso chi ha un colore della pelle diverso.

Nel racconto Curry di pollo ${ }^{16}$, invece, l'umorismo di Wadia colpisce due diversi atteggiamenti razzisti, questa volta dal punto di vista di due personaggi migranti: l'auto-razzismo, cioè una forma interiorizzata di razzismo che porta a un'accettazione passiva di stereotipi negativi sul proprio gruppo etnico di appartenenza e l'etnocentrismo.

Anandita, la protagonista del racconto, è un'adolescente di origine indiana, nata e cresciuta in Italia, che si identifica totalmente con il paese in cui vive. La ragazza vede l'India con gli occhi di un'italiana, ovvero con

$\overline{16}$ L. Wadia, «Curry di pollo» in AA. VV, Pecore nere, Roma-Bari, Laterza, p. 39-52. (CP). 
tutti i pregiudizi e gli stereotipi degli italiani nei confronti di un paese che conoscono poco.

Il bersaglio del disprezzo di Anandita è soprattutto il padre che è rimasto profondamente legato al suo paese e rifiuta di acculturarsi in Italia. Sia nell'aspetto fisico che nel comportamento, l'uomo è presentato in modo caricaturale. La caricatura e l'iperbole sono procedimenti dell'esagerazione con cui si ottengono effetti comici e servono a mettere a nudo un difetto, come emerge dalle seguenti descrizioni dell'uomo:

Mio padre, invece, in estate ed in inverno indossa lo stesso maglione bluvioletto con il collo a $\mathrm{V}$, troppo largo sulle braccia e troppo stretto sulla pancia prominente. Non ha più capelli da pettinare o oliare da un bel po' di tempo. Sebbene parli un italiano comprensibile, ragiona ancora come un contadino indiano. (CP, 40),

L'immagine dell'uomo, grassoccio e spelacchiato, suscita il riso. Ma l'impietoso ritratto del padre non si ferma qui:

[...] mio padre che si sta scolando dieci litri di tè speziato e un quintale di pane indiano con una serie di verdure asfissiate dall'olio, dalla curcuma e dai semi di senape.

[...] «Anandita!» mio padre alterna un ruggito con una serie di rutti piccanti.

[...] si scola un altro mezzo litro di tè con la grazia di un cinghiale. (CP, 42, 45, 46)

Anche la degradazione zoomorfa della figura paterna crea un effetto comico. Il termine ruggito caratterizza il modo poco umano in cui l'uomo si esprime, mentre l'associazione inconsueta e ironica tra un'animale come il cinghiale e la parola grazia ne mette a nudo un altro difetto: la sua voracità per il cibo indiano. Il riferimento a funzioni fisiologiche, «si scola» e «i rutti piccanti» ha un ulteriore effetto caricaturale e comico. In questo modo Anandita ridicolizza l'immagine del padre che rappresenta l'autorità che le vuole imporre uno stile di vita in cui lei non si vuole identificare.

Noi ridiamo di questo padre che vuole mantenere la sua indianità e che continua a lodare la cultura del suo paese. Tuttavia ci rendiamo conto che, nel caso di Anandita, il conflitto intergenerazionale, che può riguardare tutti gli adolescenti indipendentemente dal paese di origine, è aggravato dal fatto che la ragazza vuole negare una componente della sua identità perché probabilmente ha percepito che solo così può sentirsi accettata nel paese in cui vive.

In questo racconto Wadia usa il cibo anche per evidenziare il disprezzo di Anandita per l'India. Ogni volta che la ragazza menziona cibi indiani essi sono descritti con aggettivi negativi, ad esempio, come si è visto, le 
verdure sono «asfissiate dall'olio, dalla curcuma e dai semi di senape». Inoltre Anandita butta via regolarmente gli spuntini che sua madre le prepara con "pane indiano farcito di verdure strangolate nell'olio e nelle spezie» o «farcito con verdure defunte» $(\mathrm{CP}, 45,46)$. Gli aggettivi «defunte», «strangolate» e «asfissiate» che qualificano le verdure, come se fossero delle persone, hanno un effetto comico su chi legge perché sono anche un modo iperbolico con cui la ragazza esprime il suo disprezzo per un elemento come il cibo che è un marcatore dell'identità culturale. È come se Anandita, non mangiandole, volesse evitare di sentirsi strangolata e asfissiata dalle pretese del padre. Il disprezzo del proprio gruppo etnico infatti è legato a problemi di identità e come ho osservato prima, con il suo disprezzo di tutto ciò che è indiano, la ragazza vuole affermare che l'unica identità in cui si riconosce è quella italiana. Come osservano Gardaphé e Xu: «The spurning of one's ethnicity through rejection of ethnic food is an expression of self-loathing and is often engendered by a culture that degrades ethnic foodways» ${ }^{17}$.

Se, attraverso Anandita, Wadia ci dà un'immagine stereotipata dell'India vista con gli occhi degli italiani, attraverso il padre evidenzia l'etnocentrismo, cioè l'atteggiamento secondo il quale la propria cultura è l'unica o la migliore, atteggiamento che può portare a atteggiamenti xenofobi verso il paese ospitante e creare barriere. L'uomo ha vissuto per vent'anni in Italia ma è rimasto impermeabile alla cultura del paese. Wadia usa il cibo per illustrare come per quest'uomo esso rappresenti una barriera culturale, infatti continua a lodare il curry preparato dalla madre e quando vede la figlia mangiare i cereali ha una reazione di stizza:

Perché mangi queste schifezze?» mi domanda, tirando a sé la mia scodella e facendo una faccia disgustata come se l'avesse vista piena di vermi, e poco dopo l'uomo definisce i cereali «sterco di coniglio» e la pasta «tubi di gomma» $(\mathrm{CP}, 43,50)$.

Wadia ci fa ridere sui due atteggiamenti opposti della figlia e del padre, atteggiamenti che hanno effetti negativi perché impediscono da una parte il riconoscimento delle proprie origini e identità culturali e dall'altra l'inserimento nel paese in cui il migrante ha deciso di vivere. Per l'uomo l'Italia rappresenta solo il posto dove far fortuna col suo lavoro, senza tener conto che sua figlia invece ci è nata e cresciuta. In questo racconto Wadia illustra come posizioni estreme di iperidentificazione o rifiuto del paese di

17 F. L. Gardaphé e W. Xu, «Introduction: food in multi-ethnic literatures» in Melus. Special Issue. Food in Multi-Ethnic Literatures, vol. 32, n. 4, 2007, p. 6. 
destinazione possono provocare incomprensioni nell'ambito dello stesso gruppo etnico e della famiglia.

Una caratteristica di de Caldas Brito è il modo in cui coniuga l'umorismo con il fantastico in testi in cui prende posizione nei confronti di leggi migratorie xenofobe. Un primo esempio di questa commistione tra umoristico e fantastico si trova nel racconto Io polpastrello 5.42318, scritto come reazione alla Legge Bossi-Fini del 2002 che prevedeva la schedatura delle impronte degli immigrati per avere il permesso di soggiorno. Nel racconto i polpastrelli si allontanano dal corpo dei loro proprietari e vanno in questura a farsi prendere le impronte. L'elemento fantastico della separazione del dito dal corpo ha un duplice significato. Da una parte la scrittrice allude al fatto che la maggior parte di loro è impiegata in lavori manuali, dall'altra fa riferimento alla frammentazione della loro identità.

Andando tutti insieme, sono circa seimila, creano una gran confusione negli uffici della burocrazia italiana che non è preparata a un assalto così numeroso seppur pacifico. La reazione delle forze dell'ordine all'arrivo dei polpastrelli è descritta in modo umoristico in quanto i poliziotti, che rappresentano l'autorità dello stato, perdono la calma e non sanno affrontare l'emergenza. Bergson osserva che la storia del persecutore che diventa vittima della sua persecuzione è una delle situazioni tipiche dell'umorismo che in questo caso è causato da un'inversione dei ruoli ${ }^{19}$. Nel racconto di de Caldas Brito si nota questo rovesciamento tra il ruolo delle forze dell'ordine, che rinunciano al loro potere di applicare la legge, e quello dei migranti, che hanno invece il potere di gettare nello scompiglio i rappresentanti dello stato. I polpastrelli dovrebbero essere le vittime di una legge ingiusta, tuttavia si trasformano invece in persecutori delle forze dell'ordine che, alla fine disperate, per potersi liberare di loro, li mandano via.

Il bonario umorismo di questo racconto ci fa ridere ma, nello stesso tempo, ci fa riflettere su una legge discriminatoria e ingiusta e sull'importante ruolo degli immigrati nella società italiana. L'aggressione verbale e fisica a cui le forze dell'ordine sottopongono i poveri polpastrelli è contrastata con quella pacifica di quest'ultimi, che usano la loro massiccia presenza e la loro logica per sottolineare l'assurdità della legge:

Io, polpastrello 5423, balzai in avanti: «Caro questore e cari poliziotti, siamo un gruppo pacifico. Non potete trattarci così!» «Sta’ zitto!» mi gridò il

18 C. de Caldas Brito, «Io polpastrello 5.423» in Qui e là. Racconti, Isernia, Cosmo Iannone, 2004, p. 89-93. (IPO)

19 H. Bergson, op. cit., p. 95 
questore, «perché siete venuti?, cosa volete?» Come facevo a fornirgli delle spiegazioni se dovevo stare zitto? Aspettai un nuovo ordine che arrivò subito: «Parla, imbecille!» Dal basso della mia piccolezza, io, polpastrello 5.423, dissi: «Caro signor questore, sono un umile polpastrello di un immigrato, non un imbecille. Cosa vogliamo? Vogliamo rispettare la legge. Non avete ordinato che ci presentassimo in questura per lasciarvi le nostre impronte digitali? Eccoci!» (IPO, 91-92)

In questo racconto l'umorismo nasce anche dallo scarto tra il modo in cui noi lettori eravamo preparati a rappresentarci i poliziotti e il modo in cui sono presentati, in quanto sono loro i primi a disobbedire alla legge.

Il motivo della ribellione dei rappresentanti delle forze dell'ordine è ripreso in Clandestiniz ${ }^{20}$. La storia, che ha anche una dimensione fantastica, è ambientata all'aereoporto di Fiumicino dove un poliziotto semplicione (osserva Freud che il personaggio ingenuo che cerca di capire una realtà per lui nuova ha spesso un effetto comico) ${ }^{21}$ vede arrivare dei tipi molto strani: un drago sanguinante che dice di venire dall'Inghilterra e di essere in fuga da San Giorgio, un unicorno che viene dalla Francia e infine un mostro giapponese. Si tratta di personaggi un po' grotteschi la cui comicità è prevalentemente legata alla stranezza del loro corpo e all'effetto che fanno sul poliziotto. Il poliziotto li lascia entrare perché vengono da paesi non considerati a rischio, anche se l'aspetto di questi personaggi non è molto raccomandabile. Quando arrivano persone normali, gli immigrati che vogliono lavorare in Italia, il poliziotto dovrebbe fermarli perché privi del permesso di soggiorno. Alla fine li lascia entrare perché secondo lui l'Italia ha bisogno di gente che lavora e, opportunisticamente, pensa che tra di loro troverà anche chi potrà accudire sua nonna.

Il racconto è costruito sul paradosso dell'anormalità accettata e della normalità che andrebbe respinta. L'umorismo di de Caldas Brito serve a mettere in risalto gli aspetti incongrui della legge italiana. Anche in questo testo, come in quello precedente, a fare le spese dell'umorismo è la figura che dovrebbe rappresentare l'autorità: il poliziotto è presentato come ignorante e, alla fine, ribelle. Presentando questo personaggio come ignorante e ingenuo de Caldas Brito sembra inserirsi nel filone umoristico italiano delle barzellette sui carabinieri.

20 C. de Caldas Brito, «Clandestini» in El-Ghibli, n. 25, 2009. http://www.el-ghibli.provincia. bologna.it/id_1-issue_06_25-section_1-index_pos_4.html

21 S. Freud, Der Witz und seine Beziehungen zum Unbewussten, in Gesammelte Werke, Band VI, Frankfurt am Main, Fischer, 1940, p. 208. 
In questi due racconti il tema della ribellione, cioè un'infrazione alla legge, è attribuito alle forze dell'ordine italiane, secondo un procedimento di inversione che genera umorismo. In definitiva possiamo dire che in questi due racconti l'umorismo di de Caldas Brito è bonario, tuttavia è presente una componente sovversiva. Si era detto che secondo Freud l'umorismo non è rassegnato ma esprime una sfida ${ }^{22}$ e sicuramente questi racconti evidenziano l'atteggiamento ribelle della scrittrice che esprime il suo dissenso nei confronti di un ordine sociale ingiusto e rivela l'incongruità di leggi discriminanti. A questo proposito è interessante notare che l'uso del fantastico si collega all'atteggiamento di sfida di de Caldas Brito, infatti, come ha osservato Rosemary Jackson, la letteratura fantastica ha una forte componente trasgressiva ${ }^{23}$.

\section{Conclusioni}

I tre autori che ho considerato usano l'umorismo con diversi intenti. È sicuramente una strategia per rendere i loro testi piacevoli e raggiungere un pubblico ampio di lettori. Reichl e Stein usano il concetto di «community of laughter» per evidenziare che l'umorismo presuppone la condivisione di codici e di valori e ha un ruolo importante nel creare e mantenere i rapporti tra le persone di una comunità ${ }^{24}$. Anche Morreal ha osservato che l'umorismo facilita l'interazione sociale e se associato a una questione seria ne riduce l'effetto offensivo che altrimenti potrebbe avere ${ }^{25}$. Usando l'umorismo per parlare di razzismo, Lakhous, Wadia e de Caldas Brito invitano gli italiani a ridere con loro, invece di confrontarli in modo diretto. La tensione che potrebbe essere causata dalla critica di un comportamento negativo viene mitigata ed è più probabile che i lettori siano invogliati a riflettere sul loro modo di rapportarsi agli stranieri. L'umorismo così non è solo una forma di intrattenimento ma agisce anche da stimolo intellettuale.

Tutti e tre gli autori usano stereotipi e li decostruiscono rivelando come il razzismo ha le sue radici nell'ignoranza e nella paura della diversità ed anche nella rigidità mentale. La rigidità, come elemento del comico, emerge soprattutto in Lakhous e Wadia. Questo è un aspetto

22 Id., «Der Humor», cit., p. 385.

23 Cfr. R. Jackson, Fantasy. The Literature of Subversion, London, New York, Methuen, 1981.

24 S. Reichl e M. Stein, Cheeky Fictions: Laughter and the Postcolonial, Amsterdam/New York, Rodopi, 2005, p. 13.

25 J. Morreall, Taking Laughter Seriously, New York, Suny Press, 1983. 
significativo dell'analisi, perché al di là della sua portata comica la rigidità fa riferimento a un certo atteggiamento degli italiani che sono restii ad accettare i cambiamenti che stanno avvenendo nel loro paese con l'arrivo degli immigrati. Non è un caso che Wadia ricorra a personaggi anziani, in cui la rigidità è di solito più manifesta, per ridicolizzare atteggiamenti xenofobi. Nel rifiuto di far rispettare le regole de Caldas Brito non rivela tanto l'atteggiamento opposto alla rigidità, cioè la flessibilità italiana, perché la ribellione delle forze dell'ordine a leggi ingiuste non dipende da principi etici, ma dall'opportunismo. Lo stereotipo a cui fa riferimento la scrittrice brasiliana è quello dell'italiano irrispettoso della legge quando va contro i suoi interessi personali. La dimensione sovversiva dell'umorismo emerge particolarmente nei testi di Wadia e de Caldas Brito e le sue vittime sono figure di potere (il padre e le forze dell'ordine) la cui autorità, come effetto della loro ridicolizzazione, risulta sminuita.

Un altro aspetto che emerge dall'analisi è l'ignoranza degli italiani nei confronti degli stranieri che causa sovente equivoci esilaranti, come mostrano i testi di Lakhous e di Wadia ma anche che rivela la scarsa disponibilità a conoscere l'altro e come essa possa essere causa di contrasti. Si è visto, inoltre, come gli autori evidenzino atteggiamenti incongrui per denunciare l'ambiguità degli italiani verso gli stranieri che sono visti come invasori da respingere e nello stesso tempo forza lavoro di cui hanno bisogno, anche se non vogliono riconoscerlo.

In definitiva l'umorismo può svolgere un'importante funzione sociale e offrire uno sfogo per criticare la società senza aggravare contrasti che già esistono. È un modo leggero e intelligente per accattivarsi l'interesse del lettore ed è una strategia per fargli aprire la bocca al riso ma poi, nello stesso tempo, fargli inghiottire la pillola amara di alcuni aspetti tragici della migrazione come il razzismo e la xenofobia. 